International Journal of Applied Dental Sciences 2020; 6(3): 407-410

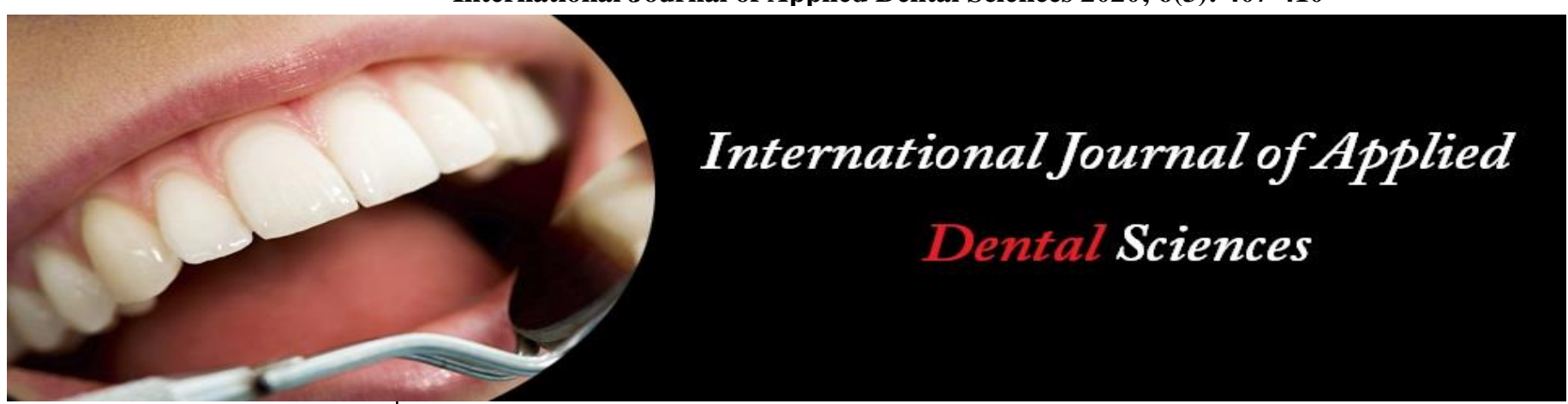

ISSN Print: 2394-7489

ISSN Online: 2394-7497

IJADS 2020; 6(3): 407-410

(C) 2020 IJADS

www.oraljournal.com

Received: 25-05-2020

Accepted: 27-06-2020

Dr. Sangita Show

MDS. Dental Surgeon,

Consultant Periodontist,

Durgapur, West Bengal, India

Dr. Arka Kanti Dey

MDS. Dental surgeon cum

Clinical tutor/Demonstrator,

Diamond harbour Medical

College and Hospital, West

Bengal, India
Corresponding Author:

Dr. Sangita Show

MDS. Dental Surgeon,

Consultant Periodontist,

Durgapur, West Bengal, India

\section{Haptics: The virtual reality in periodontics}

\author{
Dr. Sangita Show and Dr. Arka Kanti Dey
}

DOI: https://doi.org/10.22271/oral.2020.v6.i3f.985

\section{Abstract}

The simulation of clinical situations along with the acquisition of fine motor skills comprise an essential aspect of student's learning experience. However conventional approach to dental training poses certain drawbacks in terms of cost, availability, lack of real clinical cases, shortage of time, clinical supervision, and the funding of raw materials. The introduction of the concept of haptics opens the door to a more realistic clinical experience which is free from the previous limitations. Also, haptics offers the possibility of unlimited training hours by which students can gain skills without demands on manpower and resources. For the improvement of tactile sensation, the sense of touch and force-feedback might offer greater improvements to the existing learning techniques, thus enhancing the quality of education procedures. This paper provides a comprehensive insight into the domain of haptics for enhancing motor skills during clinical training of periodontal procedures.

Keywords: Technology based learning, virtual simulators, haptics, virtual reality

\section{Introduction}

Over the past decade utilization of technology based learning as well as training in both the fields of dental and medical education has increased. Medical simulation has become a valuable tool for learning and acquiring skills. In order to gain surgical expertise it takes more than merely observing patients, diagnosing and managing the disease, but needs a practical experience of the tactile information as well. Conventional clinical training is conducted in two stages. Firstly, dental students are trained on artificial teeth, placed within a manikin head, using real dental instruments, like burs, etc. Practicing on manikins reduces the risk to some extent but is less effective considering the cost, availability, and lack of rare real-world cases (cannot provide the level of detail and material properties of real life teeth and procedures). In the second stage, the students perform dental procedures on real patients under the close supervision of their professors. So, the students/clinicians go through a trial and error process by working on real patients before achieving better and more consistent experience and safe performance of dental procedures.

Despite conventional techniques have their own importance in training, students/clinicians must have a firm knowledge about the use of tools and the material properties of the organs before practicing on live patients. Hence, a system, which simulates real dental procedures graphically and haptically, might serve as a better option to increase student's knowledge/experience level and to perform mock surgeries before practicing on live patients. Introduction of haptic technology can bring about better outcomes with less error ${ }^{[1]}$.

"Haptics" refers to the area of robotics and Virtual Reality (VR) and deals with simulation devices used for sensing pressure, vibration and other senses related to touch. Dental simulator along with haptic devices allows users to touch, feel the objects and perform operations on soft and hard tissues with tactile sense ${ }^{[2]}$. Haptic device is a mechanical device which allows twoway communication or exchange of information between user and the virtual environment (Fig.1) ${ }^{[3]}$.

Desktop haptic devices with simulation software can offer the operator, a safe and an inexpensive method for improving manual dexterity or psychomotor skills in preclinical training. ${ }^{[4]}$ Simulators are capable of objective assessment offering greater level of detail such as time taken to finish the procedure, efficiency of movements and percentage of error. 


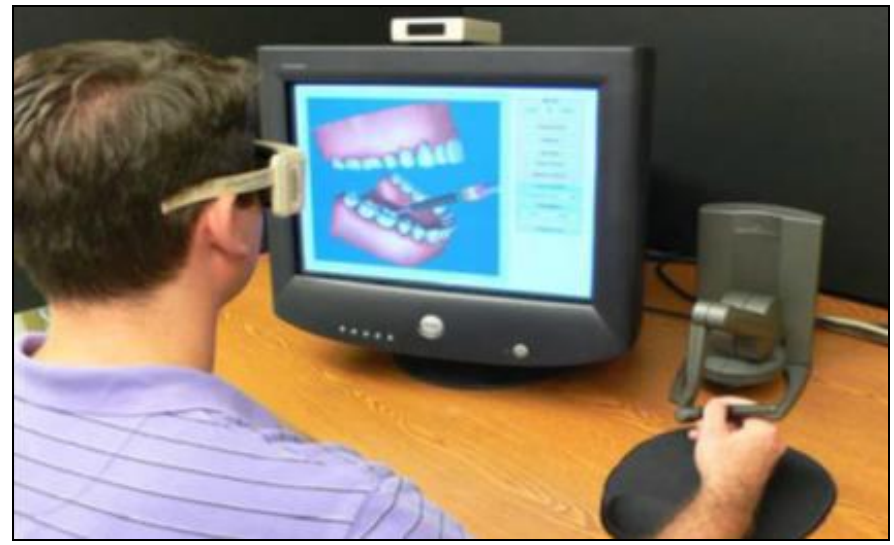

Fig 1: Simulator setup and handling the real instrument and the haptic stylus

Haptics-based simulators comprises a haptic device and a platform to facilitate dental practicing (virtual models of a human tooth or mouth). The trainee needs to hold the stylus of haptic device instead of real dental instruments and can manipulate the instruments, shown on the screen, which in turn reproduces clinical sensations in the hand of the operator through tactile feedback. The advantage of using such Virtual Reality (VR) simulators is that, they provide an opportunity for surgeons to practice surgical or operative procedures repeatedly, with no additional cost ${ }^{[3]}$.

\section{Historical Background and Principles}

- Wang et al. ${ }^{[5]}$ worked on a simulator that allows probing and cutting a virtual tooth, but the virtual tool implementation was limited to a spherical shape for simplicity.

- Kim et al. ${ }^{[6]}$ developed a dental training system with a multi-modal workbench providing visual, audio, and haptic feedback. It allows burring and drilling on the tooth with a spherical tool.

- Yau et al. ${ }^{[7]}$ proposed a dental training system utilizing material stiffness and spring force function.

- Luciano ${ }^{[8]}$ developed periosim, which allows trainee to practice diagnosing periodontal diseases that does not require deformation of tooth surface.

- Haptics works on the principles of creating virtual environment, which replaces the reality, and user can interact to perform various motor and perceptual tasks. It can help one to be mentally transported and immersed in virtual worlds through various computer softwares.

\section{Hardware for the haptic system comprises}

- Monitor and speakers

- Haptic interface device (stylus)

- Glasses and helmets for visualizing 2D video
- display as 3D

- Gloves to feel the sensations. ${ }^{[1]}$

\section{Application in Periodontics}

Differentiating between pathological and normal conditions, diagnosing and treating periodontal diseases requires skill which can be achieved by employing one of the two visuohaptic systems: periosim and a periodontal simulator ${ }^{[9,10]}$.

Periosim and periodontal simulator were developed by university of Illinois at Chicago. The application simulates three dental instruments: A periodontal probe, a scaler, and an explorer, which can be used for training students in various aspects of Periodontology.

Diagnosis: Diagnosing the periodontal disease mainly depends on probing and measuring the clinical attachment loss. Probing depth measurement vary from examiner to examiner because of variation in angulation, pressure, force etc., and thus virtual periodontal probe could be used to teach the correct probing technique, which will help in determining the health and severity of disease of periodontal tissue and thus the correct diagnosis.

Treatment: Encompasses complete elimination of etiological factors like supragingival calculus which is easily seen can be removed effectively using scalers, but the problem arises in completely removing the sub-gingival calculus, which mainly depends on tactile sensation, which can be mastered using a haptic technology while learning ${ }^{[1]}$.

In the evaluation phase, a virtual periodontal explorer may be used to determine whether the calculus has been completely removed. This evaluation could be performed with both a transparent and an opaque gingiva to contrast the results obtained by the trainee.

\section{Haptics and Bone Surgery}

In the Stanford Bio Robotics Laboratory, a visuohaptic simulation of bone surgery for training and evaluation has been devised. A hybrid data structure is used to represent the bone: A volumetric array stores the density values and attributes of the data, whereas a surface triangulation is used to render the bone graphically. The volume data and surface triangles are obtained from computer tomography or magnetic resonance data after a preprocessing procedure. The system also simulates bone dust, provides drilling sounds, and can incorporate a second haptic device as a suction and irrigation tool ${ }^{[11]}$.

Bone Navi, another computer- aided support system for implant surgery, has been developed in Japan to simulate implant placement and surgical guide fabrication for dental implant surgery

Table 1: Comparison of some of the haptic simulators available worldwide is shown in ${ }^{\text {[12] }}$

\begin{tabular}{|c|c|c|c|c|}
\hline Type of Simulators & Field & Principle & Advantage & Gap \\
\hline $\begin{array}{c}\text { Dent Sim } \\
\text { Simulator }\end{array}$ & $\begin{array}{c}\text { Tooth/cavity } \\
\text { preparation }\end{array}$ & $\begin{array}{c}\text { Infra-red emitters } \\
\text { on handpiece and } \\
\text { manikin head }\end{array}$ & $\checkmark \quad \begin{array}{l}\text { Visual feedback compared to the ideal } \\
\text { preparation. Faster evaluation though similar skill }\end{array}$ & $\begin{array}{c}\text { Restoration cannot be } \\
\text { recorded. }\end{array}$ \\
\hline $\begin{array}{c}\text { Image } \\
\text { Guided } \\
\text { Implantology }\end{array}$ & Implant & $\begin{array}{c}\text { CT scan of patient } \\
\text { (Bone) }\end{array}$ & $\checkmark$ & Patient safety: Computer guides the surgery \\
\hline $\begin{array}{c}\text { Virtual Reality Dental } \\
\text { Training System } \\
\text { (VRDTS) }\end{array}$ & $\begin{array}{c}\text { Tooth/cavity } \\
\text { preparation }\end{array}$ & $\begin{array}{c}\text { Virtual images of } \\
\text { dental instruments } \\
\text { and tooth }\end{array}$ & $\checkmark \quad$ Less costly & $\begin{array}{c}\text { Interface is in } \\
\text { the air. }\end{array}$ \\
\hline
\end{tabular}




\begin{tabular}{|c|c|c|c|c|}
\hline $\begin{array}{c}\text { Iowa } \\
\text { Dental } \\
\text { Surgical } \\
\text { Simulator }\end{array}$ & & & $\checkmark \quad$ Sense of touch through joystick & $\begin{array}{c}\text { More of haptic and less } \\
\text { of psychomotor skills }\end{array}$ \\
\hline Perio Sim & Periodontics & Periodontal probe & \begin{tabular}{|ll}
$\checkmark$ & Evaluates caries lesion and periodontal pockets \\
$\checkmark$ & Small robot like arm with force feedback system
\end{tabular} & - \\
\hline $\begin{array}{c}\text { Haptic } \\
\text { Training } \\
\text { Simulation } \\
\text { for the } \\
\text { Administration of } \\
\text { Dental } \\
\text { Anaesthesia based } \\
\text { upon } \\
\text { Accurate } \\
\text { Anatomical } \\
\text { Data }\end{array}$ & & & \begin{tabular}{|l} 
3D laser scanning, 2D data capture, data \\
processing and optimisation, 3D reconstruction of \\
objects and environments, photo-realistic \\
rendering, user interface. \\
As with the anatomical model displayed by the \\
Head and Neck Application, \\
manipulation of the virtual patient head and \\
navigation in the virtual environment are \\
supported by conventional input methods.
\end{tabular} & - \\
\hline
\end{tabular}

\section{Operating a Periodontal Simulator}

- Instrument selection: Using the control panel, one of three periodontal instruments can be selected for onscreen use: a periodontal probe, explorer, or scaler [fig. 2].

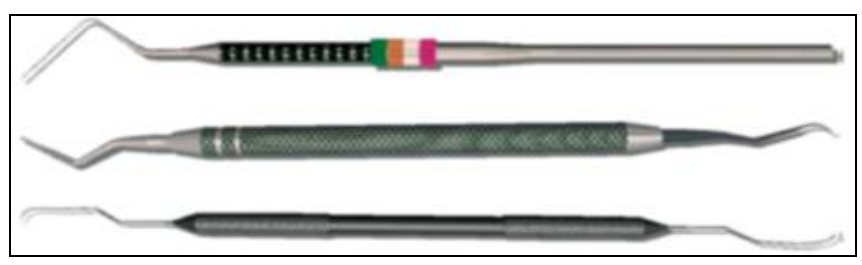

Fig 2: Virtual periodontal probe, explorer, or scaler

- Graphics Control: In the main window of the simulator, the user can see the full-screen $3 \mathrm{~d}$ model of area of interest in a dental arch along with the main control panel. The main control panel contains a variety of controls for navigation which include options to select and manipulate gingiva.

- Haptics control: Includes the basic ability to turn haptics on or off for each selected object. Also parameters such as stiffness, viscosity, static friction, dynamic friction can be controlled and altered separately for each object. By moving the haptic stylus, a trainee can move the virtual instrument on the tooth surface and feel the crevice or pocket area within the margin of the gingiva along the root surface of the tooth [figure 3,4]. The 3D virtual periodontal probe can be used to determine and measure crevice or pocket depths around the gingival margins of the teeth. The textural feel of pocket areas can be differentiated and regions of sub- gingival calculus can be located. This situation corresponds to conditions encountered clinically ${ }^{[13]}$.

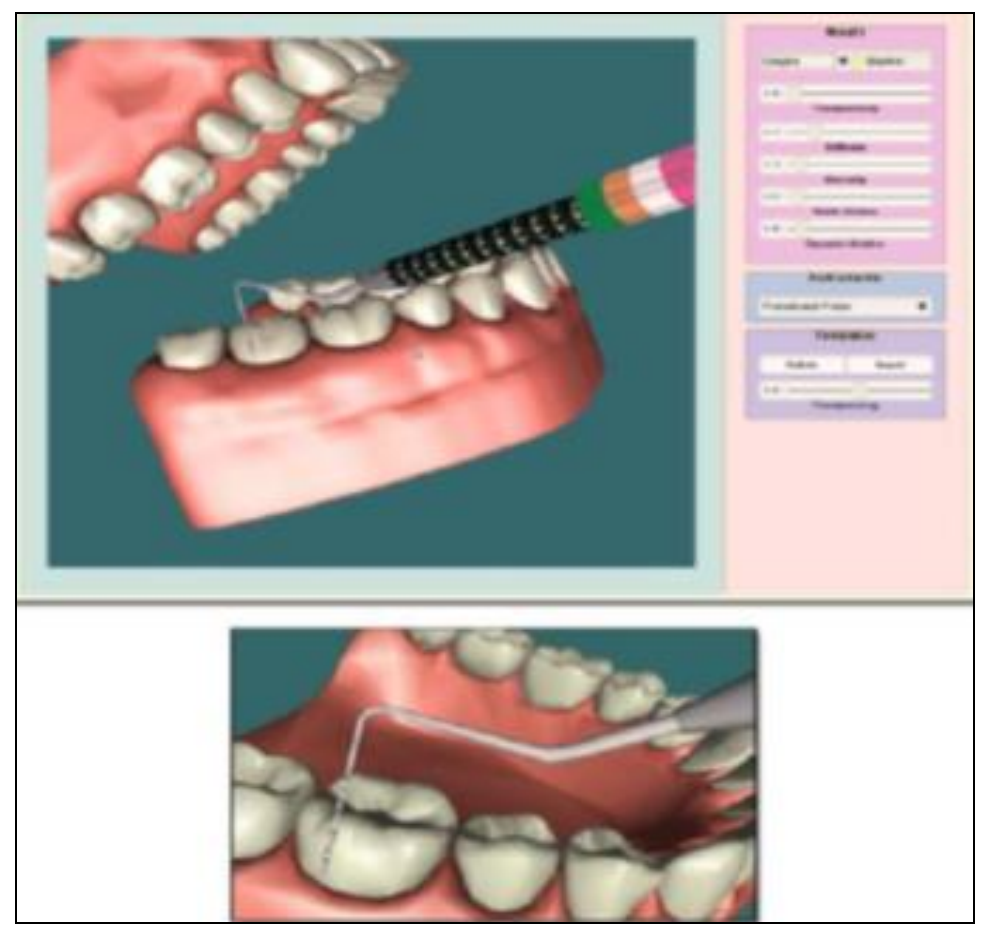

Fig. 3: Measuring pocket depth with 


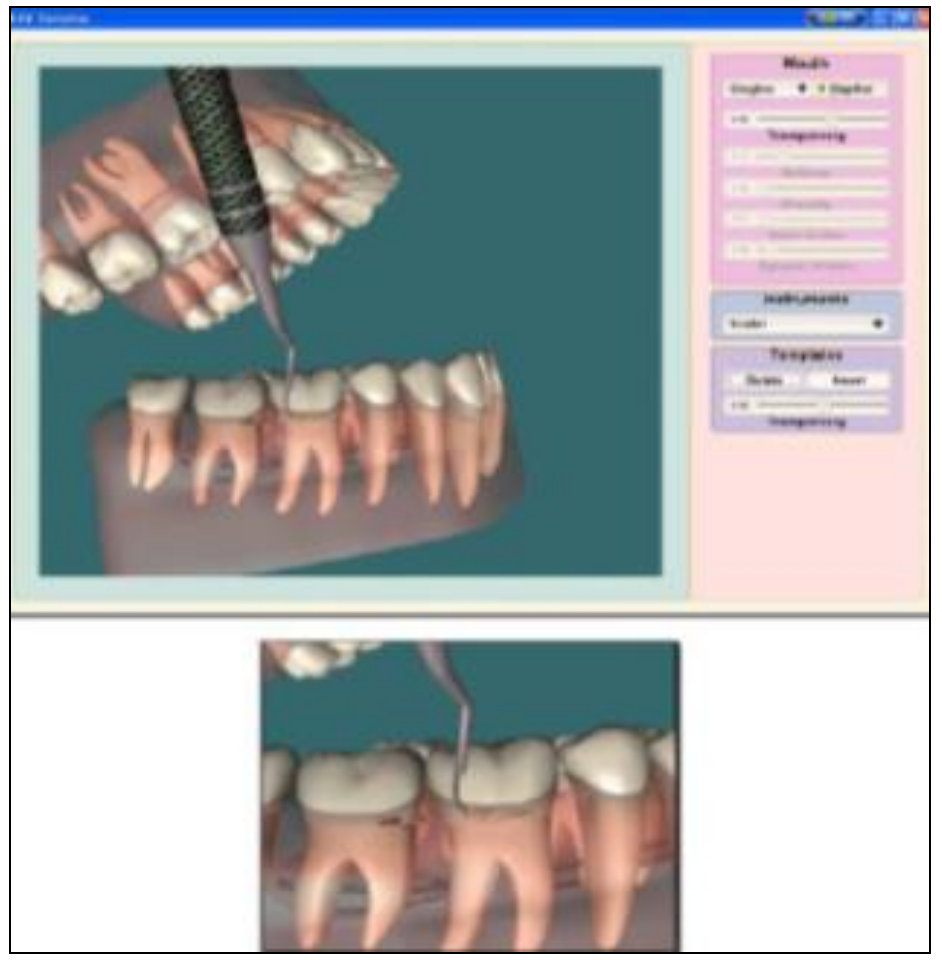

Fig 4: Calculi being removed with probe scaler

\section{Advantages}

- Reinforcement of learned dental concepts

- Correct use of dental instruments

- Correct ergonomic positioning

- Good psychomotor skills.

- Standardized evaluation

- Faster acquisition of skills

- Positive student perception.

\section{Disadvantages}

- Dental simulators are at an early or experimental stage

- Tactile perception for gingiva is not very real

- The initial cost can be substantial.

- Difficulty in maintenance and repair ${ }^{[1]}$

\section{Conclusion}

Virtual reality might establish the next level in dental education. The technology of virtual reality innovate how clinical training takes place. Unlike existing systems for clinical courses, they overcome the limitations of phantom head systems and provide standardized case, objective assessment, and interactivity. Also this methodology which improves the level of perception, sense of touch and feel and reduces the distance between the virtual and the real world and at the same time encourage a self-assessment process to identify self-directed learning. Haptics holds a promising future which might serve an excellent complementary means of training and could replace the existing ones.

\section{References}

1. Mallikarjun SA, Tiwari S, Sathyanarayana S, Devi PR. Haptics in periodontics. J Indian Soc Periodontol 2014; 18:112-3.

2. Rhienmora P, Haddawy P, Suebnukarn S, Dailey MN. Intelligent dental training simulator with objective skill assessment and feedback. Artif Intell Med [Internet]. 2011; 52(2):115-21.

3. Use of simulated learning experience in dentistry and oral health curricula. Available from: http://hwa.gov.au/sites/default/files/sles- in-dentistryoral-health-curricula- 201108.

4. Urbankova A, Engebretson SP. The use of haptics to predict preclinic operative dentistry performance and perceptual ability. J Dent Educ. 2011; 75(12):1548-57.

5. Wang D, Zhang Y, Wang Y, Lu P. Development of dental training system with haptic display. Caliimia; usa. Proceedings of the ieee international workshop on robot and human interactive communication millbrae; 2003, 159-645.

6. Kim L, hwang Y, Park SH, Ha S. Dental training system using multi-modal interface. Comput aided des appl 2005; 2:591-86.

7. Yauht, Tsou LS, Tsaimj. Octree based virtual dental training system with a haptic device. Computer aided des appl. 2006; 3:415-247.

8. Luciano CJ. Haptics-based virtual reality periodontal training simulator," master's thesis, graduate college of the University of Illinois, 2006.

9. Steinberg $\mathrm{AD}$, Banerjee P, Drummond J, Zefran M. Progress in the development of a haptic/virtual reality simulation program for scaling and root planning. J Dent Educ. 2003; 67:161-5.

10. Luciano C, Banerjee P, De Fanti T. Haptics-based virtual reality periodontal training simulator. Virtual Real 2009; 2:69-85.

11. Konukseven EI, Önder ME, Mumcuoglu E, Kisnisci RS. Development of a visio-haptic integrated dental training simulation system. J Dent Educ. 2010; 74:880-91.

12. Sivaranjani Gali, Archana Patil. The Technology of Haptics in Dental Education; Journal of Dental \& Orofacial Research, 2018, 14(2).

13. Dr. Nupur Sah, Dr. Aniket Babar, Dr. Kavita Pol Nalawade, Dr. Sneha Rohra, Dr. Purvi Sampat 5, Dr. Kajal Mahajan Haptics in Periodontics IOSR Journal of Dental and Medical Sciences (IOSR-JDMS). 2019; 18(5):28-32. 